\title{
A Genome Resource for Several North American Venturia inaequalis Isolates with Multiple Fungicide Resistance Phenotypes
}

\author{
Franz J. Lichtner, ${ }^{1,2, \dagger}$ Wayne M. Jurick II, ${ }^{2}$ Katrin M. Ayer, ${ }^{3}$ Verneta L. Gaskins, ${ }^{2}$ Sara M. Villani, ${ }^{4}$ \\ and Kerik D. Cox ${ }^{3}$ \\ ${ }^{1}$ Oak Ridge Institute for Science and Education, Oak Ridge, TN 37831 \\ ${ }^{2}$ Food Quality Laboratory, U.S. Department of Agriculture-Agriculture Research Service, Beltsville \\ Agricultural Research Center, Beltsville, MD 20705 \\ ${ }^{3}$ Plant Pathology and Plant-Microbe Biology Section, School of Integrative Plant Science, New York State \\ Agricultural Experiment Station, Cornell University, Geneva, NY 14456 \\ ${ }^{4}$ Department of Entomology and Plant Pathology, Mountain Horticulture and Crops Research \& Extension \\ Center, North Carolina State University, Mills River, NC 28759
}

\begin{abstract}
The apple scab pathogen, Venturia inaequalis, is among the most economically important fungal pathogens that affects apples. Fungicide applications are an essential part of disease management. Implementation of cultural practices and genetic sources of resistance in the host are vital components of scab management. This is the first presentation of multiple, high quality, well-annotated genomes of four North American $V$. inaequalis isolates having both sensitive and multiple fungicide resistance phenotypes. We envision that these isolates will enable investigations into fungicide resistance mechanisms, exploring fungal virulence factors and delineating phylogenomic relationships among apple scab isolates from around the world.
\end{abstract}

\section{Genomic Resource Announcement}

Understanding the genetic basis of fungicide resistance in Venturia inaequalis is important to aid resistance management strategies. Limited control options are available and the economic impact approaching 70\% crop loss can occur when an orchard becomes infected with apple scab (Frederick et al. 2014). Approximately two thirds of apples produced in the United States are consumed fresh, requiring high quality fruit devoid of surface defects. With a farm gate value estimated at nearly 4 billion dollars, the U.S. apple industry relies on blemish-free fruit for direct consumption (Jurick and Cox 2017). Fungicides are the main control strategy for scab, and prevalence of fungicide resistant populations of the apple scab fungus result in losses for growers due to control failures as well as unsalable fruit from fungal decay during storage. A sterol demethylation inhibitor (DMI) single resistant isolate, DMI/dodine (unknown mode of action/cell membrane disruption) double resistant isolate, a DMI/dodine/quinone outside inhibitor (Qol) respiration inhibitor triple resistant isolate, and a baseline sensitive isolate are the four fungicide phenotypes embodied in this sequencing project from the northeastern United States. Exploitation of fungal genomic resources will enable detailed functional information including isolates with fungicide resistance phenotypes that will be critical to augment efforts made in curating high quality whole fungal genomes, e.g., MycoCosm (Cools and HammondKosack 2013; Grigoriev et al. 2014; Nordberg et al. 2014).

\footnotetext{
${ }^{\dagger}$ Corresponding author: F. J. Lichtner; franz.lichtner@usda.gov
}

The author(s) declare no conflict of interest.

Accepted for publication 13 November 2019.

\section{Funding}

Financial support provided by Agriculture Research Service grant 8042-42430-014OOD.

\section{Keywords}

disease control and pest management, genetics and resistance, techniques 
Fungicide resistance mechanisms in a variety of fungal plant pathogens are being elucidated and genomic approaches will help identify additional mechanisms and facilitate the design of detection targets to aid in resistance management practices. For example, strobilurin (Qol) based fungicide resistance in $V$. inaequalis was first reported due to a mutation in cytochrome $b$. (Zheng et al. 2000). Sensitivity to difenoconazole, a DMI fungicide, in $V$. inaequalis was studied in orchard populations of the United States and found to be associated with increased expression of CYP51A1 as well as the occurrence of a repetitive transcriptional enhancer element (Luo and Schnabel 2008; Villani et al. 2016). Mechanism of action and detailed targets involved in dodine resistance are limited and remain unclear (Villani et al. 2015). However, our future aim is to illuminate the mode of action of dodine resistance, and further characterize difenoconazole and Qol resistance using RNAseq in future investigations, which would not be possible without the genome sequences presented here in this study.

Three isolates of $V$. inaequalis were collected from symptomatic apple leaf tissue from an orchard in Geneva, New York with a well-documented history of fungicide resistance. One baseline isolate was obtained from an orchard in Romulus, NY that had never been exposed to modern fungicides. All four isolates were single spore purified, identified using morphological and molecular methods and phenotyped for fungicide resistance as described by Villani et al. (2016). Genomic DNA was extracted using an Omega BioTek E.Z.N.A Plant DNA Kit (Omega Bio-Tek, Norcross, GA), library prep was carried out using a TruSeq PCR Free kit or TruSeq Nano kit (Illumina, Inc., San Diego, CA), and quality control was accomplished using a Qubit 4 Fluorometer to quantify the amount of DNA with a dsDNA HS kit (Thermo Fisher Scientific, Waltham, MA). Three genomes (NRRL 66904, NRRL 66905, and NRRL 66906) were sequenced by the University of Tennessee using the Illumina HiSeq2500 platform for (150 bp paired end reads, insert size of $300 \mathrm{bp}$, average read length $150 \mathrm{bp}$ ) and one genome (NRRL 66907) was analyzed using the MiSeq v3 600 bp platform via the Cornell University Biotechnology Resource Center (300 bp paired end reads, insert size of $600 \mathrm{bp}$, average read length $150 \mathrm{bp}$ ). Sequence adapter trimming and quality control were conducted with Trimmomatic and a phred score cut-off at 20. Genome assemblies were performed using SPAdes implemented with the Shovill pipeline version 1.0.0 (https://github.com/tseemann/shovill) with a minimum contig length of 1,000 and estimated genome size of $60 \mathrm{Mb}$, utilizing a reference genome including transcripts from NCBI accession QFBF00000000 and BioProject number PRJNA354841 (Passey et al. 2018). The assembled genomes ranged in size from $43.0 \mathrm{Mb}$ to $61.5 \mathrm{Mb}$ with $23 \times$ to $115 \times$ coverage. The predicted gene models through GeneMark-ES and AUGUSTUS were annotated using multiple approaches including InterproScan 5.1, SignalP 4.1, Phobius, CAZy, and antiSMASH (Almagro Armenteros et al. 2019; Bateman et al. 2017; Cantarel et al. 2009; Jones et al. 2014; Käll et al. 2007; Keller et al. 2011; Lowe and Eddy 1996; Rawlings et al. 2018; Ter-Hovhannisyan et al. 2008; Weber et al. 2015). Genomic comparisons, regarding genome size, genome completeness, numbers of transposons etc. as contained in the table, were conducted for baseline sensitive and fungicide resistant isolates.

Table 1. Functional annotation of Venturia inaequalis genomes including secondary metabolite profiles, putative secreted proteins, CAZymes, and transposable elements

\begin{tabular}{|c|c|c|c|c|}
\hline & NRRL 66904 & NRRL 66905 & NRRL 66906 & NRRL 66907 \\
\hline Fungicide phenotype & Baseline sensitive & DMI resistant & DMI/dodine resistant & DMI/dodine/Qol resistan \\
\hline Genome size $(b p) /$ coverage & $43,021,645 / 23 x$ & $48,691,399 / 50 x$ & $61,576,817 / 115 x$ & $44592039 / 54 \times$ \\
\hline Scaffolds & 6,350 & 7,555 & 6,920 & 617 \\
\hline UniProt $^{\mathrm{a}}$ & 601 & 576 & 581 & 774 \\
\hline CAZyme $^{b}$ & 346 & 350 & 346 & 396 \\
\hline BUSCO $^{\mathrm{C}}$ & $95.74 \%$ & $96.34 \%$ & $96.73 \%$ & $97.49 \%$ \\
\hline MEROPs ${ }^{f}$ & 339 & 338 & 341 & 457 \\
\hline Pfam $^{g}$ & 11,441 & 11,477 & 11,562 & 14,903 \\
\hline Transposable elements $/ \%$ masked repeats ${ }^{\mathrm{h}}$ & $48 / 1.10$ & $71 / 1.39$ & $71 / 3.76$ & $153 / 1.74$ \\
\hline
\end{tabular}

a The universal protein knowledgebase.

b Carbohydrate-Active enZymes database.

c Benchmarking universal single copy orthologs in Ascomycota.

d Signal peptide prediction program.

e Secondary metabolite clusters of orthologous groups.

f Peptidase and inhibitor database.

g Protein families' database.

$\mathrm{h}$ Transposable elements as detected through RepeatMasker database/percent genome masked as repeats. 
Functional annotation was conducted using multiple databases implemented through the Funannotate pipeline version 1.5.2 (https://github.com/nextgenusfs/funannotate). The number of scaffolds ranged from 617 to 7,555 containing 11,860 to 14,419 unique genes based on GeneMark-ES, Augustus, BUSCO (Gurevich et al. 2013). The genome completeness determined with the BUSCO v3 Ascomycota odb9 database including 1,315 targets resulting in 1,259 to 1,282 benchmarking universal single copy orthologs (Waterhouse et al. 2018). The initial gene annotations, through a Diamond blastP search of the UniProt database version 2018_09, resulted in 581 to 601 models (Buchfink et al. 2014). An analysis of the predicted secretome with SignalP resulted in 1,381 to 1,401 annotations and the number of transposable elements detected during annotation ranged from 48 to 153 (Table 1). The transposable elements included LTR-Gypsy, LTR-Pao, Helitron-3, Copia-9, BEL1, among others (Smit et al. 2015). The annotated genome includes mitochondrial genes. The differences observed here provide opportunities for further investigation into the mechanisms involved in fungicide resistance as transposons may be driving genome diversity, overall genome size, and or enabling fungicide resistance in the field under various selection pressures.

The whole genome sequencing project has been deposited at GenBank under the BioProject number PRJNA504484, and the physical strains were submitted to the USDA NRRL culture collection. Baseline sensitive isolate (NRRL 66904) BioSample number SAMN10393820, DMI single resistant isolate (NRRL 66905) BioSample number SAMN10423484, DMI/dodine dual resistant isolate (NRRL 66906) BioSample number SAMN10423485, and the DMI/dodine/Qol triple resistant isolate (NRRL 66907) BioSample number SAMN10423486.

\section{Acknowledgments}

We thank Kurt Lamour for library preparation and sequencing three of the four fungal genomes using NGS Illumina platform in his laboratory at the University of Tennessee.

\section{Literature Cited}

Almagro Armenteros, J. J., Tsirigos, K. D., Sønderby, C. K., Petersen, T. N., Winther, O., Brunak, S., et al. 2019. SignalP 5.0 improves signal peptide predictions using deep neural networks. Nat. Biotechnol. 37:420-423.

Bateman, A., Martin, M. J., O'Donovan, C., Magrane, M., Alpi, E., Antunes, R., et al. 2017. UniProt: The universal protein knowledgebase. Nucleic Acids Res. 45:D158-D169.

Buchfink, B., Xie, C., and Huson, D. H. 2014. Fast and sensitive protein alignment using DIAMOND. Nat. Methods 12:59-60.

Cantarel, B. I., Coutinho, P. M., Rancurel, C., Bernard, T., Lombard, V., and Henrissat, B. 2009. The Carbohydrate-Active EnZymes database (CAZy): An expert resource for glycogenomics. Nucleic Acids Res. 37:D233-D238.

Cools, H. J., and Hammond-Kosack, K. E. 2013. Exploitation of genomics in fungicide research: Current status and future perspectives. Mol. Plant Pathol. 14:197-210.

Frederick, Z. A., Villani, S. M., Cooley, D. R., Biggs, A. R., Raes, J. J., and Cox, K. D. 2014. Prevalence and stability of qualitative Qol resistance in populations of Venturia inaequalis in the Northeastern United States. Plant Dis. 98:1122-1130.

Grigoriev, I. V., Nikitin, R., Haridas, S., Kuo, A., Ohm, R., Otillar, R., et al. 2014. MycoCosm portal: Gearing up for 1000 fungal genomes. Nucleic Acids Res. 42:D699-D704.

Gurevich, A., Saveliev, V., Vyahhi, N., and Tesler, G. 2013. QUAST: Quality assessment tool for genome assemblies. Bioinformatics 29:1072-1075.

Jones, P., Binns, D., Chang, H. Y., Fraser, M., Li, W., McAnulla, C., et al. 2014. InterProScan 5: Genome-scale protein function classification. Bioinformatics 30: 1236-1240.

Jurick, W. M., II, and Cox, K. D. 2017. Chapter 14: Pre- and postharvest fungal apple diseases. Pages 371-380 in: Achieving Sustainable Cultivation of Apples. K. Evans, ed. Burleigh Dodds Science Publishing, Cambridge, U.K.

Käll, L., Krogh, A., and Sonnhammer, E. L. L. 2007. Advantages of combined transmembrane topology and signal peptide prediction-the Phobius web server. Nucleic Acids Res. 35:429-432.

Keller, O., Kollmar, M., Stanke, M., and Waack, S. 2011. A novel hybrid gene prediction method employing protein multiple sequence alignments. Bioinformatics 27: 757-763.

Lowe, T. M., and Eddy, S. R. 1996. TRNAscan-SE: A program for improved detection of transfer RNA genes in genomic sequence. Nucleic Acids Res. 25:955-964.
Luo, C. X., and Schnabel, G. 2008. The cytochrome P450 lanosterol $14 \alpha$-demethylase gene is a demethylation inhibitor fungicide resistance determinant in Monilinia fructicola field isolates from Georgia. Appl. Environ. Microbiol. 74:359-366.

Nordberg, H., Cantor, M., Dusheyko, S., Hua, S., Poliakov, A., Shabalov, I., et al. 2014. The genome portal of the Department of Energy Joint Genome Institute: 2014 updates. Nucleic Acids Res. 42:D26-D31.

Passey, T. A. J., Armitage, A. D., and Xu, X. 2018. Annotated draft genome sequence of the apple scab pathogen Venturia inaequalis. Microbiol. Resour. Announc. 7: e01062-18. http://mra.asm.org/lookup/doi/10.1128/MRA.01062-18

Rawlings, N. D., Barrett, A. J., Thomas, P. D., Huang, X., Bateman, A., and Finn, R. D. 2018. The MEROPS database of proteolytic enzymes, their substrates and inhibitors in 2017 and a comparison with peptidases in the PANTHER database. Nucleic Acids Res. 46:D624-D632.

Smit, A. F. A., Hubley, R., and Green, P. 2015. RepeatMasker Open-4.0. http:// www.repeatmasker.org

Ter-Hovhannisyan, V., Lomsadze, A., Chernoff, Y. O., and Borodovsky, M. 2008. Gene prediction in novel fungal genomes using an ab initio algorithm with unsupervised training. Genome Res. 18:1979-1990.

Villani, S. M., Biggs, A. R., Cooley, D. R., Raes, J. J., and Cox, K. D. 2015. Prevalence of myclobutanil resistance and difenoconazole insensitivity in populations of Venturia inaequalis. Plant Dis. 99:1526-1536.

Villani, S. M., Hulvey, J., Hily, J.-M., and Cox, K. D. 2016. Overexpression of the CYP51A1 gene and repeated elements are associated with differential sensitivity to DMI fungicides in Venturia inaequalis. Phytopathology 106:562-571.

Waterhouse, R. M., Seppey, M., Simao, F. A., Manni, M., loannidis, P., Klioutchnikov, G., et al. 2018. BUSCO applications from quality assessments to gene prediction and phylogenomics. Mol. Biol. Evol. 35:543-548.

Weber, T., Blin, K., Duddela, S., Krug, D., Kim, H. U., Bruccoleri, R., et al. 2015. AntiSMASH 3.0-A comprehensive resource for the genome mining of biosynthetic gene clusters. Nucleic Acids Res. 43:W237-W243.

Zheng, D., Olaya, G., and Koller, W. 2000. Characterization of laboratory mutants of Venturia inaequalis resistant to the strobilurin-related fungicide kresoxim-methyl. Curr. Genet. 38:148-155. 